\title{
A Novel Framework for Guaranteeing Quality of Service in Wireless Sensor Networks: Design, Simulation and Evaluation
}

\author{
Omar Said \\ Information Technology Department, College of Computers and Information Technology \\ Taif University, Taif, Saudi Arabia
}

\begin{abstract}
Recently, Wireless Sensor Networks (WSN) became one of the most important areas of research in the world. The Quality of Services (QoS) is the cornerstone of WSN efficiency due to providing the network with all requirements for efficient sending and receiving of data. Because of WSN different infrastructures, there are different QoS parameters required for this environment. In this paper, a general framework to guarantee the WSN QoS is proposed. The proposed framework aims to produce a standard solution for WSN QoS problems. How to solve the problems of traditional protocols, taking into account the compatibility, which may result from a collection of individual solutions in one general framework, is the paper target. Also, the proposed framework adds new strategies like congestion prediction and network management technologies to outperform the previous solutions. Finally, our framework is simulated using network simulation package (NS2) and compared with individual solutions to make sure that it is more robust and efficient, which leads us to standardization.
\end{abstract}

\section{Keywords}

Wire Sensor Networks, Network Management, QoS, Wireless Communication, Network Simulation.

\section{INTRODUCTION}

It is well known that wired networks use end-to-end communication. Hence, the parameters like bandwidth, delay, and loss can provide good QoS. In WSN, these parameters are not fully applicable because sensor nodes don't use end-to-end communication. Each node communicates only with its neighboring nodes and no connection needs to be established between source and destination. In addition, intermediate sensor nodes generate data during routing, along with the most challenging problem which is energy and this can be considered another problem. So, parameters like coverage, exposure, energy cost and network life time should be raised to be new QoS parameters. The coverage problem may happen due to location and network management. Exposure provides measures of how an object can be observed by a sensor over a period of time. Energy cost finds the best route to destination as regards energy conservation. Network life time is the total working time of WSN until it is not able to satisfy user's needs [1], [2]. To face these challenges, a powerful management system for WSN should be constructed provided that this system considers the critical parameters such as WSN node power degree, WSN bandwidth, and WSN instrumentations.
This paper proceeds as follows; in Section 2, the related works are introduced. In Section 3, the proposed system is discussed. In Section 4, simulation and results are showed. Finally, the conclusion is introduced in Sections 5.

\section{REALTED WORKS}

The Proposed Transport Protocol for Reliable Data Transfer in WSN proposed by F. Yunus [3] is much close related work. The basic idea of this trial is to propose a transport layer protocol to handle the reliability and congestion control issues. The main shortcoming in this protocol is neglecting some important QoS parameters such as bandwidth, standardization, Density, and others.

\section{OUR WSN MANAGEMENT SYSTEM}

\subsection{System components}

The system consists of four components as follows; the General sink node, the Manager sink node, the Classifier sink node, and the Prioritization sink node. For the connection of these components, see Fig.1.

\section{- The General sink node}

In our proposed framework, the General sink can be considered as the top layer. The General sink node is used to receive the cumulative reports from the lower layer. These reports contain information which scales the throughput of entire WSN. Upon these reports the General sink node can take a decision regarding some problems such as congestion and delay. The General sink node should have strong specs such as big buffer size, high central processing units, and long power live. This is because it should accomplish more functions in our management system. These functions are; collection of reports which are received from the lower layer sink nodes, analysis of these reports, run decision making algorithms, direction of each sink node, and solving the problems which may occur within the WSN sessions.

\section{- The Manager sink node}

The Manager sink node is the first component in the second layer of our proposed management system. The Manager sink is considered as a head of sensors cluster. The job of this sink node is to collect reports sent from the sensors in its cluster. Hence, it analyzes these reports and merges them in one report. Consequently, it should send this collective report to the General sink node in the upper layer. The main specs, which should be required in the Manager sink node, are sufficient buffer space and powerful central processing unit (but not as powerful as the General sink node). 


\section{- The Classifier sink node}

The Classifier sink node is considered as the second component in the second layer of our proposed management system. This sink node is found behind the Manager sink node. The job of the Classifier is to rearrange and mark the information of sensors which are located in its Manager sink node area. Also, it should have an ability to differentiate between each type of data (i.e. multimedia, text, or image). The specs of the Classifier sink node are high buffer capacity and suitable processing power.

\section{- Prioritization sink node}

The Prioritization sink node is considered as the third component in the second layer of our proposed management system. The job of this node is to determine the first data which should be transmitted specially in case of WSN starvation. The specs of this node are high buffer capacity and powerful processing unit. This node works on the data received from the Manager sink node report.

\subsection{How the system works}

Our system comprises three management layers; the General sink node layer, the Multi-Functional sink layer, and the Sensors layer. Our proposed WSN management system is considered as bi-directional due to the messages, sent to and received from the system components. The first direction is from the Sensors layer to the Multi-Functional layer. In this direction the data, which are collected by sensors, is sent to the Multi-Functional layer components. Each component in this layer handles this data beginning from the Manager sink node, passing through the Classifier and Prioritization sink nodes, and ending with the Manager sink node. Hence, the Manager sink node sends this data to the General sink node layer. The other direction starts with the General sink node layer through the Multi-Functional layer down to the Sensors layer.

To clarify how our proposed system works the two directions should be studied intensively. The first direction consists of two connection types. One from the lower level layer (the Sensor layer) and the other from the MultiFunctional layer to the General sink node layer. The sensors are clustered into groups and send the collected environmental data to the Manager sink node. Hence, the Manager collects and filters the sensors messages. The Manager filtering process separates the correct data from the damage data which decreases the WSN overload. Correspondingly, it collects them in one report. The filtering process uses the technique stated in [4]. The Manager sink node sends a copy from its cumulative report to the Classifier sink node. The Classifier node signs each data depending on its type. Hence, easily, it can separate each data type in one group, which helps the entire system to send special required information in specific time. To accomplish this job, the classifier uses the technique in [5]. Furthermore, the Manager sink node sends a copy from its report to the Prioritization sink node. The prioritization sink node determines the data which should be sent firstly. These data can be determined as regards some parameters such as the WSN throughput, available component power, and importance of transmitted data which can be defined from the system feedback process. The prioritization system which is introduced at [6] can be used to help in accomplishing this job. The second connection type is from the Manager sink, the Classifier sink and the Prioritization sink node to the General sink node. The Manager sink sends its report to the General sink node to describe each sensor state, which is determined using some parameters such as level of each sensor power, congestion time and MAC address, bandwidth consumption, and notable data type which should be sent from this area. Also, the Classifier sink node sends its report to the General sink node. This report contains the state of the collected data which describes type, size, and history of this data. Moreover, the Prioritization sink node sends its report which contains prioritized data. The prioritization report contains the most important data, followed by the least important data.

The second direction is an opposite one which starts when the General sink node receives reports of the MultiFunctional layer. The General sink node starts to analyze these reports using the technique which is introduced at [7]. The General sink node should scale the throughput of each WSN cluster using some parameters such as bandwidth, the size of transmitted data, the delay, and the congestion. Also, these parameters should be compared with the sensors power capacity. It's well known that the sensor power limitation is an extremely challenge in the WSN. The General sink node sends a report to the Manager sink node informing it with the descriptive state of its WSN cluster sensors (whether it is in sleep or active mode). Hence, the Manager sink node should send a simple message to the sensors specifying the state of each one. The special case of this direction is fired when an urgent event occurs such as critical power levels or bottlenecks. So, a special report should be sent from the General sink node to the Manager sink node containing the description of urgent event with the steps which should be followed to solve the resulting problems.

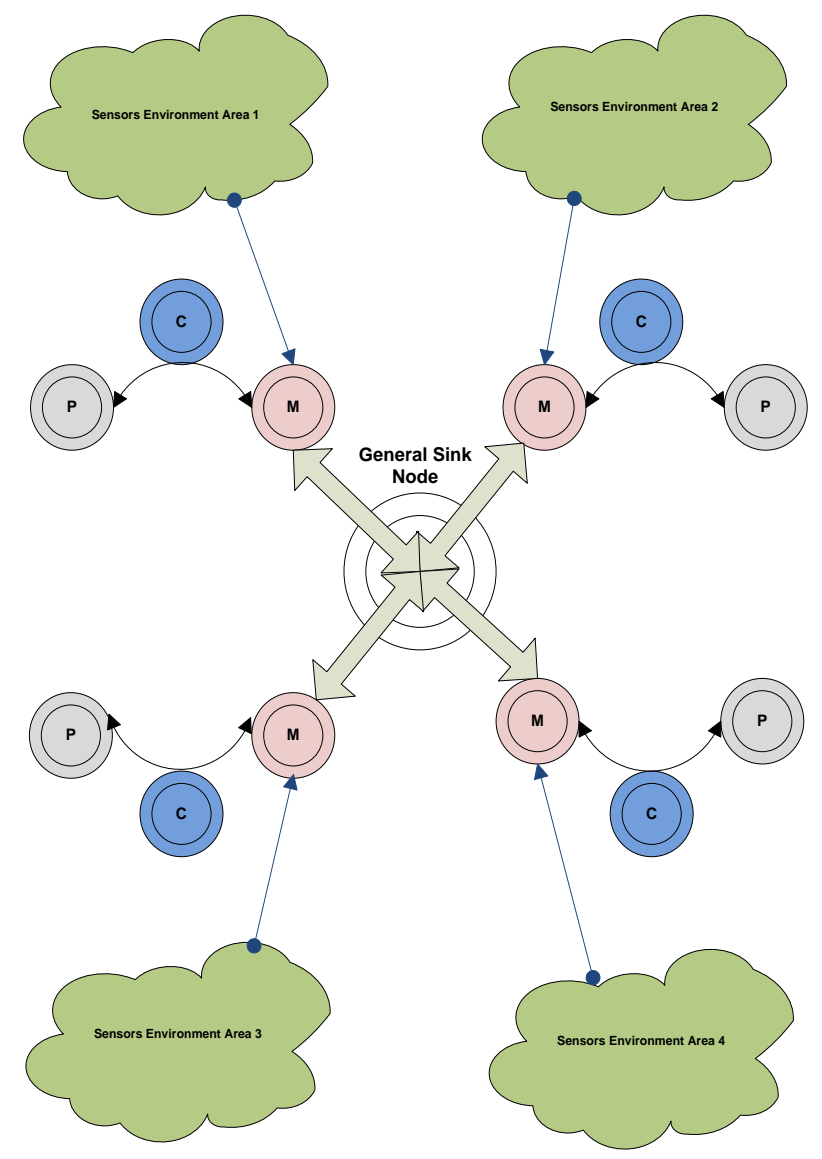

Fig. 1: General view of the proposed system

\subsection{Proposed system advantages}

- It is aware with up-to-date changes in the WSN.

- It gives more than one strategy to deal with WSN different states. 
- It contains General sink node which adapts the entire WSN by awareness with its different states.

- It is flexible because it minimizes or maximizes the transmitted information.

- It is a fault tolerant system due to its recovery technique.

- It is a scalable system due the clustering system infrastructure.

\section{SIMULATION AND EVALUATION}

\subsection{Simulation setup}

A simulation environment is constructed using the Network Simulation package (NS2) [8]. This environment is used to test the proposed WSN network management system and compare it with a current (normal) WSN management system [9]. In our simulation, the WSN is divided into multiple groups. Each group contains number of sensors. Each group is responsible for gathering the required information about its environment. The information, which is used in our simulation, is multimedia, images, and text. The multimedia data uses the jpeg extension. The image data uses the jpg extension. The sensor nodes are distributed in square area. The management sink nodes (General, Manager, Classifier, and Prioritization) are located in the center of sensors nodes in each group. Furthermore, each node should be defined as a compound module containing Radio, Application, MAC, Routing, and Management Strategy. The definition of protocols and parameters in our simulation is necessary because it declares the simulation results. The simulation parameters are listed in the Table 1.

Table 1: Simulation parameters

\begin{tabular}{|l|l|}
\hline \multicolumn{1}{|c|}{ Simulation Parameters } & \multicolumn{1}{c|}{ Value } \\
\hline Simulation Time & $500 \mathrm{sec}$ \\
\hline Number of Sensors & 200 to 300 \\
\hline Number of Networks & 20 \\
\hline Collusion & Yes \\
\hline Dynamic WSN & Yes \\
\hline Simulation field & $126 \mathrm{~m} * 126 \mathrm{~m}$ \\
\hline MAC protocol & TMAC \\
\hline Routing protocol & Multipath routing protocol \\
\hline Mobile object Inter-arrival & 5 \\
\hline Sensor deployment & Uniform / Grid \\
\hline Packet rate & $250 \mathrm{kbps}$ \\
\hline Maximum MAC & frame size 2500 \\
\hline Radio bandwidth & $25 \mathrm{MHz}$ \\
\hline Modulation Type & PSK \\
\hline
\end{tabular}

In order to evaluate the performance of our simulation, some metrics should be introduced to describe supposed WSN configurations. These configurations should be the same as real world WSN applications in addition to simulation-based studies. Moreover, the related attributes and variables, which are used in our simulation, together with the simulation metrics should be predicted behaviors of our WSN topology which is described in Fig. 2. The simulation performance parameters are in the following:

- Throughput: one of the performance metrics which should be calculated at each sink node. Throughput is the number of the packets successfully received at the sink node divided by the total number of the packets sent to the sink node including any retransmissions.

- Latency: is the amount of time which is needed to transfer one packet from one sensor to the General sink node. The latency performance parameter is calculated at the application level. There are some variables which affect the latency parameter. These variables are network size, topologies and collision models.

- Total transmitted data to the General sink node: there are two levels of this metric. The first level refers to total amount of number of packets which have been sent to the Manager sink node by the sensor nodes. The second level refers to the total amount of number of packets which have been sent to the General sink node by the Manager sensor nodes. In other words, it refers to the amount of time taken by the sensor nodes to detect the required environment information and report to the sink node.

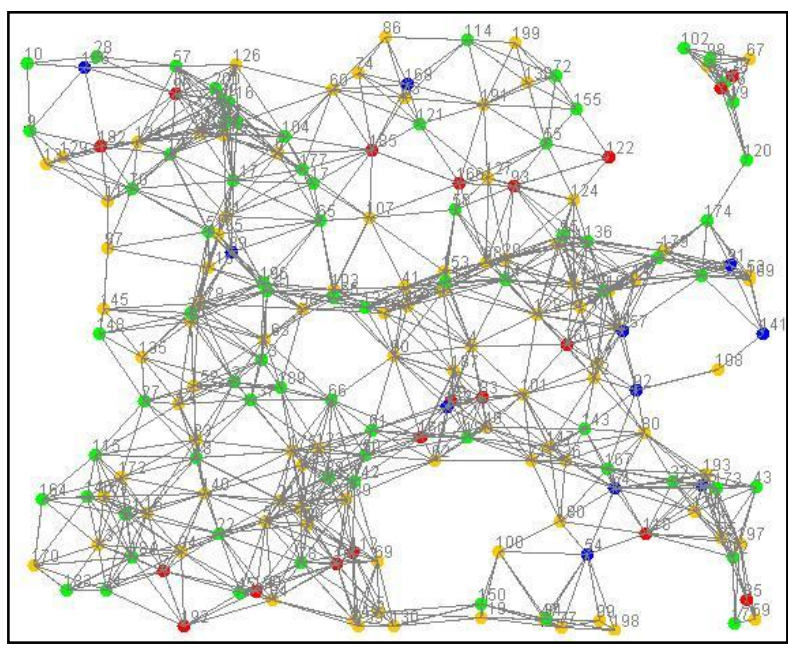

Fig. 2: General View of our simulation model with sensor distribution on multi-environment

\subsection{Results and discussion}

The throughput of the network is high because the network management system divides the WSN into groups. Each of these groups has a well defined area. The simulation results in Fig. 3 prove this claim. The fluctuations of throughput in our management system are minor and less than one in current management system. However, our system throughput has some drawbacks which can be stated as follows; the throughput is expected to decrease gradually if the number of nodes is increased. But the results, which are provided in Fig. 3 for various numbers of nodes till 100 nodes, don't prove this note exactly. Furthermore, the collision which is done when the number of nodes equals 130 causes a dramatic decrease in the throughput.

In our simulation, the end-to-end latency under different number of nodes with manager sink is measured. In our management WSN system, majority of the packets which should reach to the sink manager node needs more time. This delay is done when the number of nodes increases. This note can be interpreted using a fact "by increase in the number of nodes, the packets, which are transmitted through more servers to reach the destination, should be delayed". Fig. 4 shows that increase in the number of nodes means most of the packets reach the sink with delay.

In the simulation system, the number of nodes increases means the total data transmission measured at the manager sink node increases. Fig. 5 illustrates this fact. 


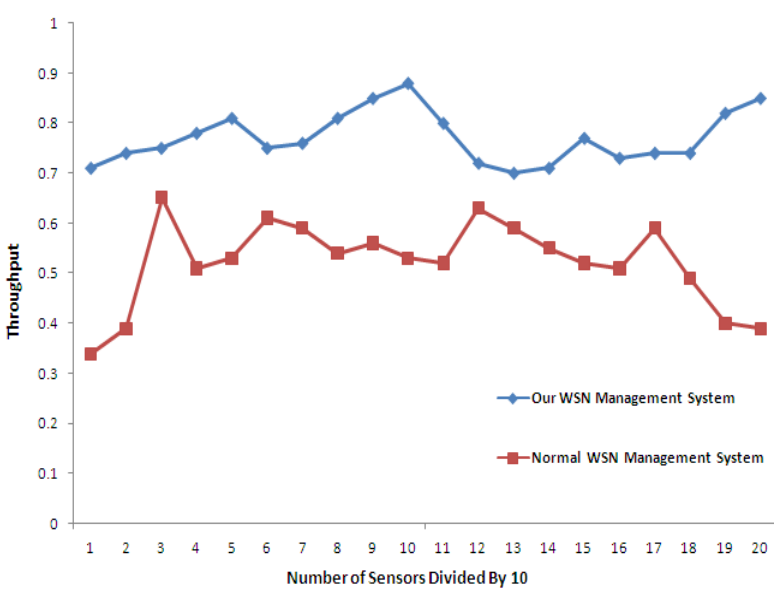

Fig. 3: The WSN throughput.

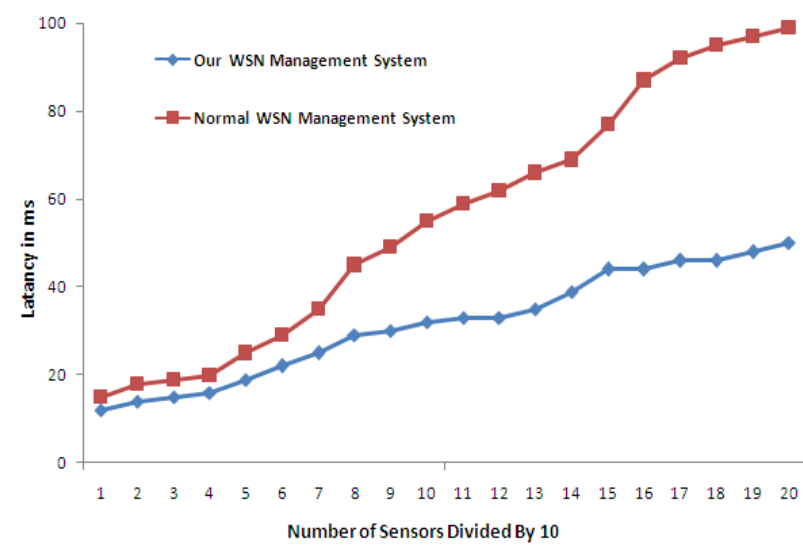

Fig. 4: The WSN latency.

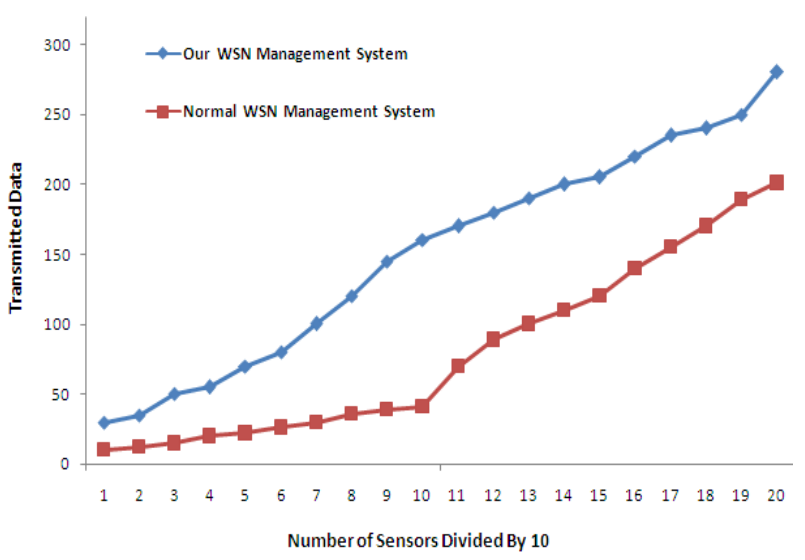

Fig. 5: The total transmitted data from the General layer to the Sensors layer and via versa.

\section{CONCLUSION}

In this paper, a novel framework to manage a WSN and gurantee the QoS such as bandwidth, delay, and jitter is introduced. The proposed framework consists of three layers; the General sink node layer, the Multi-Functional layer, and the Sensors layer. The basic idea of our proposed framework is to distribute management processes on multiple components. Our simulation proves that the proposed system enhanced the normal WSN system in the following parameters, the WSN throughput, the total transmitted data, and the lost packets. Also, the results showed that the effieincy of each management component is between $89.2 \%$ and 99.1. In addition, the WSN is adpative system because it can change its stratgy in case of sudden event occurrence. Furthermore, our management system is sclable because its infrastructure is based on the clustering strategy. Finally, our system can be considered as a long term soultion for the WSN QoS.

\section{REFERENCES}

[1] Chen, D., and Varshney, P. K. QoS support in wireless sensor networks: A survey. In Proc. of the 2004 International Conference on Wireless Networks (ICWN 2004) (Las Vegas, Nevada, USA, June 2004), vol. 1, pp. 227-233.

[2] Liqiang Tao, Fengqi Yu. A Novel Congestion Detection and Avoidance Algorithm for Multiple Class of Traffic in Sensor Network. Proceedings of the IEEE International Conference on Cyber Technology in Automation, Control, and Intelligent Systems, Kunming, China, Pages: 72-77, March 20-23, 2011.

[3] Ahmed Ayadi, Energy-efficient and reliable transport protocols for wireless sensor networks: state-of-art. Wireless Sensor Network, March, vol. 3, n 3, pp. 106113, 2011.

[4] Seung Tae Hong, A New Data Filtering Scheme Based on Statistical Data Analysis for Monitoring Systems in Wireless Sensor Networks, IEEE 13th International Conference on High Performance Computing and Communications (HPCC), Banff, Alberta, Canada, PP: 635 - 640, 2-4 Sept. 2011.

[5] Tsagkatakis, G., Dictionary based reconstruction and classification of randomly sampled sensor network data. IEEE 7th Conference Sensor Array and Multichannel Signal Processing Workshop (SAM), NJ, USA, PP:117 - 120, 17-20 June 2012.

[6] Potdar, V. ; Rathnayaka, A.J.D., Prioritizing Information for Achieving QoS Control in WSN, 24th IEEE International Conference on Advanced Information Networking and Applications (AINA), Perth, Australia, PP: 835 - 842, 20-23 April 2010.

[7] Xin Song, Cuirong Wang, A data analysis and visualization algorithm based on locally linear approximating for WSN, IEEE International Conference on Networking, Sensing and Control (ICNSC), PP: $507-$ 511, IL, USA 10-12 April 2010.

[8] The Network Simulator - ns-2, 2008, http://www.isi.edu/nsnam/ns/

[9] Abdulmaowjod, A., Mohammed, H.M. Simulation \& performance study of wireless sensor network (WSN) using MATLAB. 1st International Conference on Energy, Power and Control (EPC-IQ), PP: 307 - 314 Nov. 30 2010-Dec. 2010 